\title{
A Simple Analytical Model for RObust Header Compression in Correlated Wireless Links
}

\author{
Romain Hermenier, Francesco Rossetto, Matteo Berioli \\ Institute of Communications and Navigation, German Aerospace Center (DLR) \\ Oberpfaffenhofen, 82234 Weßling, Germany \\ E-mail: \{romain.hermenier, francesco.rossetto, matteo.berioli\}ddlr.de
}

\begin{abstract}
RObust Header Compression (ROHC) has been successfully included in some wireless standards in order to reduce the excessive IP overhead for small packets, for instance Voice over IP frames. So far, there is limited understanding on how the ROHC performance depends on the design parameters and the characteristics of the wireless channel. In this paper we propose an analytical model that provides simple expressions for the probability of losing synchronization as a function of the mentioned parameters, and also yields insightful relationships between the design variables and the desired system performance. The results are validated against sophisticated and realistic models of ROHC.
\end{abstract}

\section{INTRODUCTION}

The importance of ROHC for wireless systems is undisputed [1]-[6], since it enables to compress many IP headers by over an order of magnitude with respect to their original size. Such scheme can be very important for instance for Voice over IP wireless systems, whose payloads are small and thus large IP/UDP/RTP headers would generate an intolerable overhead. The key property of ROHC is the capability to resist to larger packet error rates than classic header compression schemes, which is a necessary virtue in wireless links. The main trick is the capability to recover the transmitted header even if up to $W$ consecutive packets have been lost.

There is a quite large amount of simulation studies on the effectiveness of ROHC in literature [1]-[3], which extensively investigates the performance of $\mathrm{ROHC}$ with respect to many metrics of interest (delay, jitter, error probability, etc.). There has been some work that attempted to explore from an analytical point of view the performance of ROHC [3]-[5]. These analytical studies have shed some light into the behavior of ROHC, but rarely do they provide simple mathematical expressions and hence it is hard to infer the qualitative dependence between important design parameters (like $W$ or the timeouts that govern ROHC) to the characteristics of the wireless channels. Moreover, previous work [3]-[5] focused on channels which cause independent losses. While a memoryless channel is a useful starting point, wireless channels are often correlated and therefore a problem analysis for correlated channels is just as necessary.

In this work we address the previous points with a focus on the ROHC U-mode that is the most relevant for short packets (e.g., VoIP). After an introduction on ROHC in Section II, we provide in Section III an analytical model that, under some simplifications, enables to get insightful, first order, closedform expressions for the probability that the decompressor may lose synchronization with the compressor, which will be denoted as out-of-synchronization probability and will be the main performance metric in this paper. Therefore, our formulae clearly show the dependency between the channel characteristics, the out-of-synchronization probability and the design parameters. Moreover, we study the performance of ROHC with a Gilbert-Elliot channel [7], which is a simple yet useful and widespread model for correlated wireless channels at packet level. The model is validated in Section IV against more refined simulations of $\mathrm{ROHC}$ and show that indeed the qualitative trends for ROHC performance are correctly captured. Finally, Section V reports the conclusions.

\section{SySTEM MODEL}

ROHC is a header compression scheme for Internet protocols, which was developed and standardized by the IETF in 2001 [6]. It was conceived to reduce the header sizes of IP packets to be sent through a cellular link, which is characterized by high packet loss rate and residual bit errors.

\section{A. ROHC State Machine}

To achieve a high compression efficiency, ROHC uses state machines at the compressor and decompressor sides. Those state machines are based on the fact that ROHC classifies the fields of a packet header into two categories: the static one (such as IP addresses) and the dynamics one (such as Timestamp). The compressor state machine is composed of three states, namely the Initialization and Refresh (IR), the First Order (FO) and the Second Order (SO) states (Fig. 1). In the IR state packets are sent uncompressed (with additional bytes for the context identifier), whereas in the FO only the dynamic fields and the context identifier are transmitted uncompressed (the static fields are omitted). Finally in SO state an encoded version of the dynamic fields as well as the dynamics fields that changed are sent, resulting in a header of a couple bytes. At the decompressor side, the state machine is also composed of three states (Fig. 2). After the successful decompression of the first received IR packet, the decompressor creates the context by storing the header information for this specific identifier. This context will be used to reconstruct the following compressed headers and is updated with the header information after each successful decompression. The decompressor switches therefore from the No Context (NC) state to the Full Context (FC) state, where all kinds of ROHC packets (IR, FO, SO) can be decompressed. The last remaining state of the decompressor state machine is the Static Context (SC). Its utility will be explained later on in this section. 


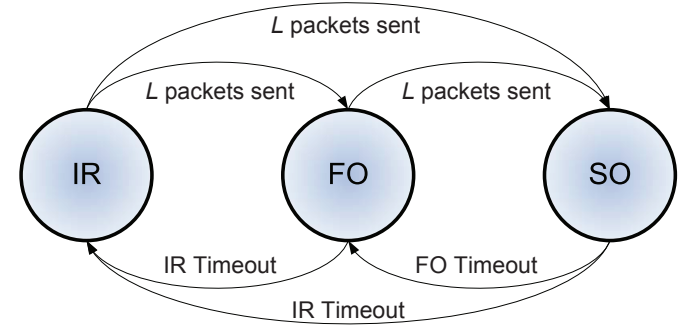

Fig. 1. ROHC state machine of the compressor in U-mode

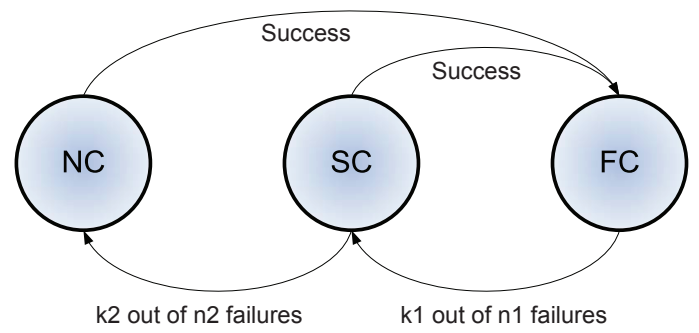

Fig. 2. ROHC state machine of the decompressor in U-mode

Moreover ROHC can operate in three different modes of operation: Unidirectional (U), Bidirectional Optimistic (O) and Reliable (R). The major difference between these three modes is how the state transitions are handled and the lack of a feedback channel for the U-mode. Since the focus is on the U-mode within this paper, we refer to [6] for a detailed description of the $\mathrm{O}$-mode and R-mode.

In U-mode, the compressor starts in IR state and sends $L$ packets before switching forward to the next compression level (Fig. 1). These $L$ packets are transmitted to establish the context at the decompressor side and $L$ usually depends on the link characteristics such as the round trip time. In order to come back to initial compression levels, the U-mode uses two timeouts: the IR timeout (IRT) to switch downward from the SO or FO state to the IR state and the FO timeout (FOT) to come back from the SO state to the FO one. These two timeouts are used to ensure context synchronization between the compressor and decompressor since no feedback channel is considered in U-mode. Depending on the configuration of these three parameters ( $L$, IRT, FOT) the compression efficiency may vary: low values of $L$, associated with high values of FOT and IRT, will increase the compression efficiency since the compressor remains longer in the most compressed state (SO). On the other hand, a high value of $L$ together with short timeouts ensures a more error resilient context synchronization, albeit at the cost of a lower compression efficiency.

Regarding the decompressor, if all received packets are successfully decoded, it stays in the FC state (Fig. 2). The decompressor switches from FC to SC only if $k_{1}$ packets out of the last $n_{1}$ received packets have been unsuccessfully decoded (CRC failed). In this intermediate state the decompressor can only decode IR or FO packets. Therefore if it receives one of them and the decompression is successful, it moves back to the FC state. However, if over the last $n_{2}$ received packets, $k_{2}$ had a CRC failure, the decompressor moves downward to the NC state, where it will wait for an IR packet (all other received packets in this state are dropped).

\section{B. ROHC Robustness}

The most important feature of the ROHC protocol is its robustness. ROHC minimizes the error propagation by using a Window-based Least Significant Bits (W-LSB) encoding scheme, which enhances its robustness. W-LSB is defined by an interpretation interval $\left[-p, 2^{k}-1-p\right]$ of size $2^{k}$, where $k$ represents the $k$ least significant bits of the encoded field value and $p$ the offset with respect to the previously received field value (we refer to [4], [6] for more details about the WLSB scheme). Since field values undergoing small negative changes are not considered here, this mechanism ensures that the decompressor is still able to work properly even if up to $\left(2^{k}-1-p\right)-1$ packets are lost consecutively [8].

This robustness is enhanced by the use of the LSB wraparound algorithm [6], applied when too many consecutive packets are lost (bigger than $\left(2^{k}-1-p\right)-1$ ). In such a case the decompressor shifts the interpretation interval of $2^{k}$ and tries to decode the received field value with the new interpretation interval. If the decompression succeeds, the decompressor updates the context but waits two successfully decompressed packets before delivering the next frame to the upper layers. Otherwise, the $k_{1}$ out of $n_{1}$ rule is applied. ${ }^{1}$

Thus, if more than $\left(2^{k}-1-p\right)-1+2^{k}$ packets are lost in a row, the decompressor does not manage to decode the next arriving SO packet and is said to be out-of-synchronization. When the receiver is out-of-synchronization and in FC or SC state, the reception of an IR or FO packet enables to retrieve the synchronization, whereas in the NC state the decompressor only updates its context with an IR packet.

For the rest of this work, we define $W$ as being the maximal number of packets that can be lost in a row without losing the context synchronization. $W$ is expressed as follows:

$$
W=\left(2^{k}-1-p\right)-1+2^{k}
$$

Out of this description of the ROHC protocol and using a Gilbert-Elliott channel, we derived a realistic Markov chain (model 1), which is fully compliant with the ROHC standard and which allows us to estimate the out-of-synchronization probability $\left(P_{\mathrm{OoS}}\right)$. Depending on the configuration, model 1 may have thousands of states, which is not really practical to handle and does not provide deep insight into the protocol behavior. In the following section we will see that by means of some simple assumptions, a chain of few dozen states can be defined (model 2), which yields a precise and useful approximation of the system. In this paper the simpler model 2 is described, while the results of the accurate and realistic model 1 are provided to validate the outcome of the other model.

\section{MODEL DERIVATION}

In order to characterize the system, three elements must be modelled: the compressor, the channel, and the decompressor.

\footnotetext{
${ }^{1}$ Since only an erasure channel is considered, we voluntarily omit the algorithm to repair an incorrect sequence number update as defined in [6]
} 
The model described within this section (model 2) is based on a set of assumptions, which aims to reduce the complexity of model 1 while still correctly predicting the qualitative trends of the protocol performance against the design parameters. These assumptions are listed hereafter:

- ROHC mode: The focus is here on the ROHC U-mode, because it is the most widespread and practically relevant for short packets (e.g., VoIP).

- Channel model: A Gilbert-Elliott erasure channel is considered. This channel is modeled by a two state Markov Chain: the good state $\mathrm{G}$ (correct reception of the packet) and the bad state B (the packet is lost and the upper layers are not aware that a packet was sent). Let us define as $P_{\mathrm{X}, \mathrm{Y}}$ the transition probability from state $\mathrm{X}$ to $\mathrm{Y}$. The transition matrix is uniquely determined by $P_{\mathrm{G}, \mathrm{B}}$ and $P_{\mathrm{B}, \mathrm{G}}$, which are inversely proportional to the average time spent in the good and bad state, respectively. The GilbertElliott channel is also equivalently defined by the average duration of a sequence of bad states $L_{\mathrm{B}}=1 / P_{\mathrm{B}, \mathrm{G}}$ and the average erasure probability $\epsilon=P_{\mathrm{G}, \mathrm{B}} /\left(P_{\mathrm{G}, \mathrm{B}}+P_{\mathrm{B}, \mathrm{G}}\right)$ [7].

- Compressor: The FO packets are not taken into account for this model because of their limited actual impact. Moreover, $L=1$ for the sake of simplicity. The compressor state machine comprises only two states: IR and SO states. Moreover, it is assumed that the compressor decides the type of the packet independently in every slot between IR and SO. An IR frame is generated with probability $P_{\mathrm{IR}}$, and therefore IR packets are sent with a memoryless, geometric backoff with average value:

$$
\mathrm{IRT}=\frac{1}{P_{\mathrm{IR}}}
$$

By using these probabilities, the IRT is no longer deterministic and becomes geometric. Thus the knowledge of the compression level of the previous packets is not required anymore. We remark that the assumption of a geometric rather than deterministic backoff has already been used in other network models to yield more tractable formulae (see for instance the analysis of 802.11 systems [9] or the ALOHA stability [10]). The qualitative trends of the system are still correctly predicted, while the numerical performance is often about the same up to a multiplicative constant. It will be proved in the results section that it is indeed the case also in this setting.

- Decompressor: Since no FO packets are considered, the SC state of the decompressor is omitted as well. Therefore the decompressor state machine is composed of two states (the NC and the FC states) and works as follows:

- If no packets are lost, the decompressor remains in FC state and works properly.

- If $W$ or less than $W$ packets are lost due to channel impairments, the decompressor is still able to decode the next SO packets thanks to the use of the LSB wraparound algorithm.

- However if the decompressor realises that more than $W$ packets are lost, there is a major context damage and the decompressor cannot decompress the next arriving packet. Thus it switches back directly to the NC state and waits for an IR packet. Neither the $k_{1}$ out of $n_{1}$ nor the $k_{2}$ out of $n_{2}$ rules are considered, as in [6]. Until the correct reception of an IR packet, the decompressor is out-of-synchronization.

The decompressor loses synchronization if more than $W$ packets in a row have been lost and after this event one SO packet is received. We remark that the last condition is important: if the decompressor never received packets, it would never be aware that the synchronization has been lost.

While the decompressor is synchronized, the model tracks the number $w$ of consecutively lost packets. Thus the model developed here is a Markov Chain in which $W+1$ states track the value of $w, 0 \leq w \leq W$. If more than $W$ packets have been corrupted by the channel, the decompressor may not yet be aware of the loss of synchronization and the chain remains in a state " $W^{+}$" until a packet is correctly delivered by the physical layer (note that in this situation the channel must have transitioned from the bad to the good state). If the packet is an IR frame, the node retrieves synchronization and returns to the $w=0$ state. Otherwise, the decompressor realises it has lost synchronization and moves into a $(\mathrm{OoS}, \mathrm{G})$ state, where the $\mathrm{G}$ represents the channel condition. The chain remains in the $(\mathrm{OoS}, \mathrm{G})$ until either the channel transitions into the B state (and the chain moves into $(\mathrm{OoS}, \mathrm{B})$ ) or an IR packet is received, and hence the decompressor recovers the synchronization and can return to the $w=0$ state. The decompressor has lost synchronization when it is in either the $(\mathrm{OoS}, \mathrm{G})$ or $(\mathrm{OoS}, \mathrm{B})$ state.

Fig. 3 depicts this Markov Chain model and the corresponding transition probabilities.

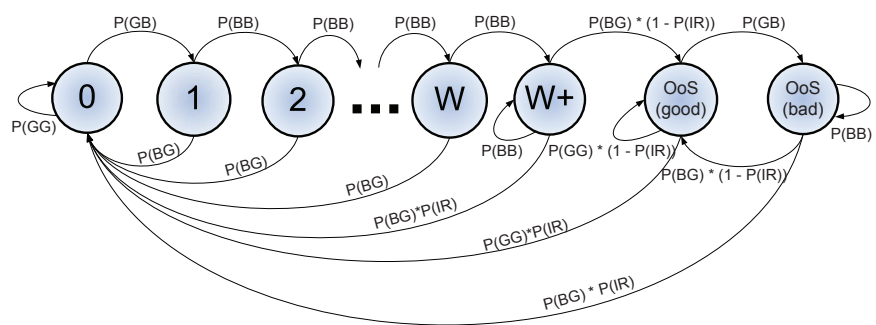

Fig. 3. Markov Chain model for the ROHC modeling in U-mode

Let us denote by $\pi_{w}, \pi_{W^{+}}, \pi_{\mathrm{OoS}, \mathrm{G}}, \pi_{\mathrm{OoS}, \mathrm{B}}$ the steady state probabilities of state $w, W^{+}$and of the two out-ofsynchronization states, respectively. After some straightforward but tedious analytical steps, $\pi_{0}$ can be evaluated as:

$$
\begin{aligned}
\pi_{0} & =\frac{1}{1+F_{W}+F_{\mathrm{OoS}}} \\
F_{W} & =\frac{\sum_{w=1}^{W} \pi_{w}+\pi_{W+}}{\pi_{0}}=\frac{P_{\mathrm{G}, \mathrm{B}}}{P_{\mathrm{B}, \mathrm{G}}} \\
F_{\mathrm{OoS}} & =\frac{\pi_{\mathrm{OoS}, \mathrm{G}}+\pi_{\mathrm{OoS}, \mathrm{B}}}{\pi_{0}}= \\
& =\frac{P_{\mathrm{G}, \mathrm{B}}}{P_{\mathrm{B}, \mathrm{G}}}\left(P_{\mathrm{B}, \mathrm{B}}\right)^{W}\left(P_{\mathrm{B}, \mathrm{G}}+P_{\mathrm{G}, \mathrm{B}}\right) \frac{1-P_{\mathrm{IR}}}{P_{\mathrm{IR}}}
\end{aligned}
$$

Therefore, $P_{\mathrm{OoS}}$ is equal to: 


$$
\begin{aligned}
P_{\mathrm{OoS}} & =\pi_{\mathrm{OoS}, \mathrm{G}}+\pi_{\mathrm{OoS}, \mathrm{B}}= \\
& =\frac{\frac{P_{\mathrm{G}, \mathrm{B}}}{P_{\mathrm{B}, \mathrm{G}}}\left(P_{\mathrm{B}, \mathrm{B}}\right)^{W}\left(P_{\mathrm{B}, \mathrm{G}}+P_{\mathrm{G}, \mathrm{B}}\right) \frac{1-P_{\mathrm{IR}}}{P_{\mathbb{R}}}}{1+\frac{P_{\mathrm{G}, \mathrm{B}}}{P_{\mathrm{B}, \mathrm{G}}}\left(1+\left(P_{\mathrm{B}, \mathrm{B}}\right)^{W}\left(P_{\mathrm{B}, \mathrm{G}}+P_{\mathrm{G}, \mathrm{B}}\right) \frac{1-P_{\mathrm{IR}}}{P_{\mathrm{IR}}}\right.}(6)
\end{aligned}
$$

Eq. (6) is not particularly insightful but can be simplified under reasonable hypothesis in realistic settings. First of all, it shall be assumed that $\epsilon \ll 1 \rightarrow L_{\mathrm{B}} \ll L_{\mathrm{G}} \rightarrow P_{\mathrm{G}, \mathrm{B}} \ll$ $P_{\mathrm{B}, \mathrm{G}}$. This means that the channel does not introduce too many errors (say, below 10\%). Hence, the denominator of Eq. (6) is very close to (just slightly larger than) 1 . Moreover, the IR timeout will be assumed to be much larger than 1 (otherwise, uncompressed packets are sent too often and the ROHC efficiency is too low), thus $P_{\mathrm{IR}} \simeq 0$. The numerator can be approximated as:

$$
\begin{aligned}
P_{\mathrm{OoS}} & \simeq \frac{P_{\mathrm{G}, \mathrm{B}}}{P_{\mathrm{IR}}}\left(1-P_{\mathrm{B}, \mathrm{G}}\right)^{W}=\frac{1}{L_{\mathrm{G}}}\left(\frac{L_{\mathrm{B}}-1}{L_{\mathrm{B}}}\right)^{W} \mathrm{IRT} \simeq \\
& \simeq \frac{\epsilon}{L_{\mathrm{B}}}\left(1-\frac{1}{L_{\mathrm{B}}}\right)^{W} \mathrm{IRT}
\end{aligned}
$$

The expression links the two parameters that describe the Gilbert-Elliott channel $\left(\epsilon\right.$ and $L_{\mathrm{B}}$ ) and the two ROHC design parameters $W$ and IRT with the out-of-synchronization probability, which is our main metric.

A natural question is how to pick the value of $W$ so that $P_{\mathrm{OoS}} \ll \epsilon$, that is to say, how to design the system so that the out-of-synchronization probability does not significantly worsen the intrinsic error rate of the channel. Let us define as $A$ the ratio $P_{\mathrm{OoS}} / \epsilon$ and let us set $A \gtrsim 0$ (in practice, $A<0.1$ ). Hence:

$$
W=\frac{\log \left(\frac{A L_{\mathrm{B}}}{\operatorname{IRT}}\right)}{\log \left(1-\frac{1}{L_{\mathrm{B}}}\right)}
$$

If in addition $L_{\mathrm{B}} \gg 1$ :

$$
W \simeq \log \left(\frac{\mathrm{IRT}}{A L_{\mathrm{B}}}\right) L_{\mathrm{B}}
$$

This equation formally proves an intuitive fact: in the Gilbert-Elliott channel, the maximum number of packets that can be lost in a row should be proportional to the burst length $L_{\mathrm{B}}$. Similar reasoning for the IRT yields:

$$
\mathrm{IRT}=A L_{\mathrm{B}}\left(1+\frac{1}{L_{\mathrm{B}}-1}\right)^{W}
$$

Eqs. (7), (9) and (10) provide simple and intuitive relationships between the system and environment parameters.

\section{Numerical Results}

Unless otherwise stated, the Gilbert-Elliott model parameters adopted are $\epsilon=2 \%$ and $L_{\mathrm{B}}=5$, which are reasonable values for terrestrial wireless channels [7]. Moreover, the transmitter generates IPv6/UDP/RTP headers and the SO headers length is $5 \%$ of the IR header size.

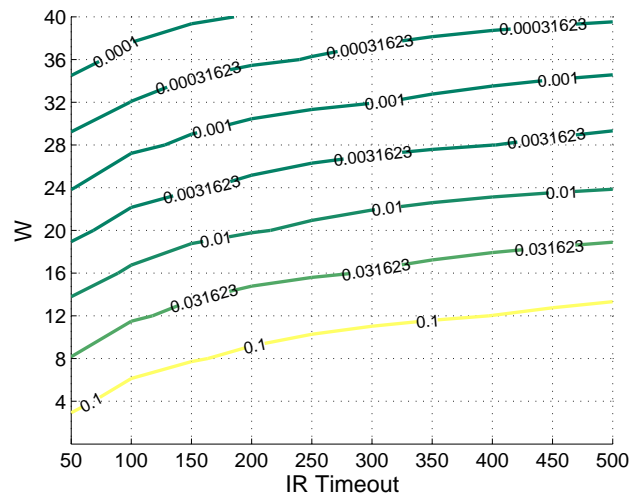

Fig. 4. Contour plot of $P_{\mathrm{OoS}}$ computed according to model 2. $L_{\mathrm{B}}=5$ and $\epsilon=2 \%$. The labels through the curves correspond to the $P_{\mathrm{OoS}}$.

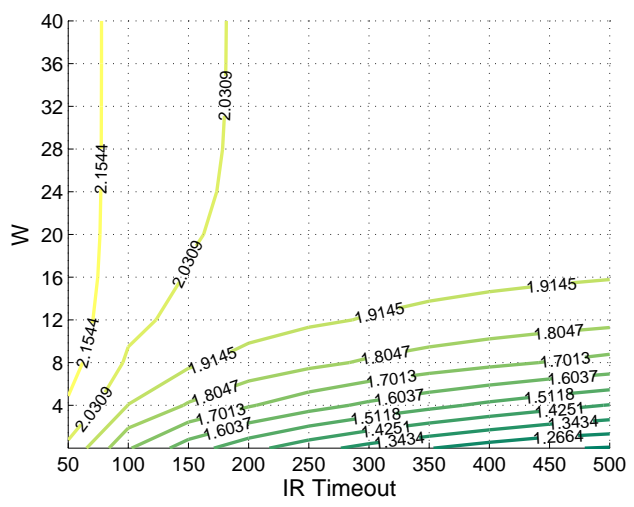

Fig. 5. Contour plot of the ratio of $P_{\mathrm{OoS}}$ for model 2 and $P_{\mathrm{OoS}}$ for model 1. $L_{\mathrm{B}}=5$ and $\epsilon=2 \%$. The labels through the curves is $P_{\mathrm{Oos}}$.

The first metric of interest is the $P_{\mathrm{Oos}}$ (Eq. (6)) against the two design parameters ( $W$ and IRT), which is depicted in Fig. 4. The contour lines are computed for logarithmically spaced values of $P_{\mathrm{OoS}}$ and they confirm that $P_{\mathrm{OoS}}$ is very sensitive to the value of $W$ (it decays exponentially with it), while it is not as deeply impacted by the IRT. For conventional IR Timeouts of 300 [11], the value of $W=13$ [8] without wraparound would yield a rather high $P_{\mathrm{Oos}}$ of about $6.5 \%$, which is not acceptable in most wireless systems. Instead, the wraparound mechanism that extends $W$ to 29 enables to reach a definitely more tolerable $0.25 \%$. Hence, in correlated wireless channels, the wraparound is necessary if a large IRT and hence a high efficiency should be attained.

Fig. 5 shows the ratio between $P_{\mathrm{OoS}}$ for model 2 and the same quantity for model 1 . It is clear that in a vast range of values the simplified model correctly predicts $P_{\mathrm{OoS}}$ up to a multiplicative number that is rather insensitive of the actual values of $W$ and IRT and is quite close to 2 . Hence, the simpler model 2 enables to get a quick estimate of $P_{\mathrm{OoS}}$ for a first order system design.

Figs. 6 and 7 show an example of how the simplified model can help to tune the system. In both cases $\epsilon=2 \%$ and the goal is to size $W$ or IRT, respectively, so as to ensure that $P_{\mathrm{OoS}} \leq$ $\epsilon / 10$ as the burst length $L_{\mathrm{B}}$ is changed, that is to say the overall 


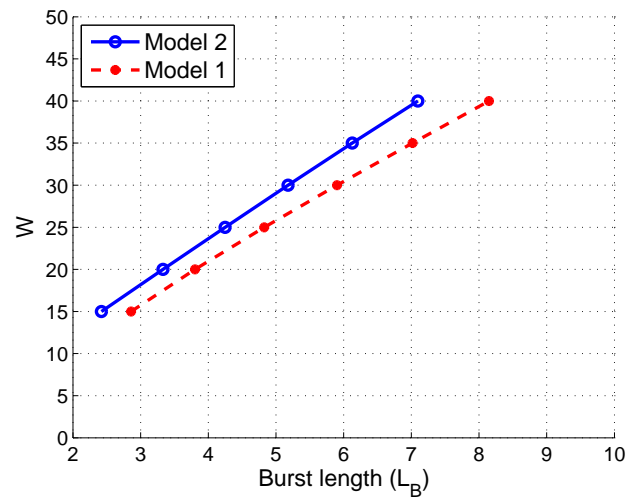

Fig. 6. Value of $W$ that guarantees $P_{\mathrm{OoS}} \leq \epsilon / 10=0.2 \%$ against $L_{\mathrm{B}}$.

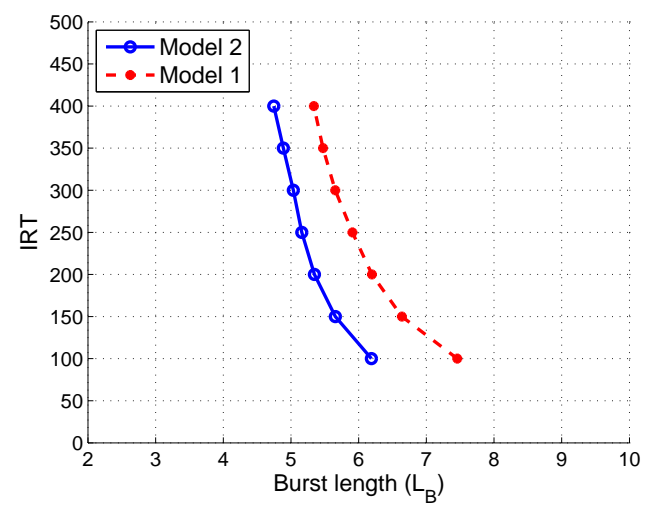

Fig. 7. Value of the IR Timeout that guarantees $P_{\mathrm{OoS}} \leq \epsilon / 10=0.2 \%$ against $L_{\mathrm{B}}$.

packet error rate is not significantly affected by the ROHC loss of synchronization. For the reference value of IRT $=300$, Fig. 6 shows the minimum value of $W$ for this purpose for both models. It is clear how the simplified model provides a handy and accurate first order estimate of $W$. Moreover, the linear relationship between $L_{\mathrm{B}}$ and $W$ is confirmed (see Eq. (9)). Fig. 7 depicts the maximum IRT for $W=29$ so as to ensure this quality of service constraint. Note that the IRT predicted by model 2 is about half of the actual value. This factor of two is in agreement with the discrepancy between the two models shown in Fig. 5. The qualitative dependence between IRT and $L_{\mathrm{B}}$ in Eq. (10) is also validated.

Finally, Fig. 8 shows the amount of average reduction of the header size due to ROHC as in the setting of Fig. 7. ROHC enables, as expected, a reduction of the header sizes of about 20 times. Moreover, once again model 2 offers a first order yet quite accurate prediction of the system behavior.

\section{CONCLUSIONS}

This paper has proposed a simple analytical model that provides clear and intuitive formulae for the ROHC behavior in the Gilbert-Elliott channel. This model provides easy to understand relationship between the design parameters and the channel characteristics and provides useful first order formulae to set the $W$ and IRT parameters.

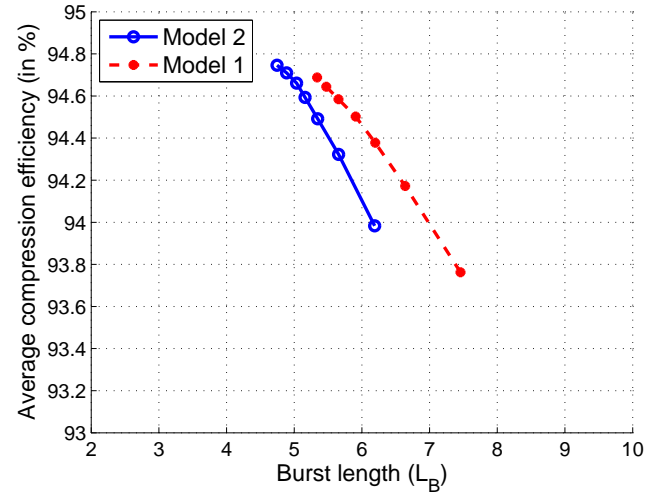

Fig. 8. Average bandwidth efficiency of ROHC for an IPv6/UDP/RTP header against $L_{\mathrm{B}}$ when $W=29$ and the IRT is chosen as in Fig. 7.

Directions of future work are the extension of the model for the $\mathrm{O}$ - and R-mode.

\section{ACKNOWLEDGMENTS}

The research leading to these results has been partially funded by the European Community's Seventh Framework Programme (FP7/2007-2013) under Grant Agreement $\mathrm{n}^{\circ}$ 233679. The SANDRA project is a Large Scale Integrating Project for the FP7 Topic AAT.2008.4.4.2.

\section{REFERENCES}

[1] S. Rein, F. Fitzek, and M. Reisslein, "Voice quality evaluation in wireless packet communication systems: a tutorial and performance results for rhc," Wireless Communications, IEEE, vol. 12, no. 1, pp. 60 - 67, Feb. 2005.

[2] D. Taylor, A. Herkersdorf, A. Doring, and G. Dittmann, "Robust Header Compression (ROHC) in Next-Generation Network Processors," Networking, IEEE/ACM Transactions on, vol. 13, no. 4, pp. 755-768, aug. 2005.

[3] H. Wang, J. Li, and P. Hong, "Performance analysis of ROHC U-mode in wireless links," Communications, IEE Proceedings-, vol. 151, no. 6 , pp. 549-551, dec. 2004.

[4] C. Y. Cho, Y. H. Chew, and W. K. G. Seah, "Modeling and analysis of robust header compression performance," in IEEE WoWMoM, Taormina (Italy), Jun. 2005.

[5] A. Couvreur, L.-M. Le Ny, A. Minaburo, G. Rubino, B. Sericola, and L. Toutain, "Performance Analysis os a Header Compression Protocol: The ROHC Unidirectional Mode," Telecommunication Systems, vol. 31, no. 1, pp. 85-98, Jan. 2006.

[6] C. Bormann, C. Burmeister, M. Degermark, H. Fukushima, H. Hannu, L.-E. Jonsson, R. Hakenberg, T. Koren, K. Le, Z. Liu, A. Martensson, A. Miyazaki, K. Svanbro, T. Wiebke, T. Yoshimura, and H. Zheng, Robust Header Compression (ROHC): Framework and four profiles: RTP, UDP, ESP, and uncompressed, IETF RFC 3095 Std., July 2001.

[7] L. Badia, N. Baldo, M. Levorato, and M. Zorzi, "A markov framework for error control techniques based on selective retransmission in video transmission over wireless channels," IEEE J. Select. Areas Commun., vol. 28, no. 3, pp. 488-500, Apr. 2010.

[8] B. Wang, H.-P. Schwefel, K.-C. Chua, R. Kutka, and C. Schmidt, "On implementation and improvement of robust header compression in umts," in IEEE PIMRC, 15-18 Sept. 2002.

[9] F. Calì, M. Conti, and E. Gregori, "Dynamic tuning of the IEEE 802.11 protocol to achieve a theoretical throughput limit," IEEE/ACM Trans. Networking, vol. 8, no. 6, pp. 785-799, Dec. 2000 .

[10] S. S. Lam, "Packet switching in a multi-access broadcast channel with application to satellite communication in a computer network," Ph.D. dissertation, Department of Computer Science, University of California, Los Angeles, Mar. 1974.

[11] A. Minaburo, L. Nuaymi, K. D. Singh, and L. Toutain, "Configuration and analysis of robust header compression in umts," in IEEE PIMRC, sept. 2003. 